\title{
EFECTOS DE POLÍTICAS COMERCIALES EN LA DISTRIBUCIÓN DEL INGRESO Y ASIGNACIÓN DE RECURSOS, EN UN MODELO DE EQUILIBRIO GENERAL*
}

\author{
JaIme Serra Puche \\ El Colegio de México
}

\section{INTRODUCCIÓN}

LA PRÁctica de políticas comerciales origina efectos sobre los precios de los bienes que se desea proteger y, a través de cambios en precios relativos, la asignación de recursos y su distribución, están sujetos a cambios. El análisis de estos cambios debe llevarse a cabo dentro de un modelo de equilibrio general si interesa estudiar los efectos derivados de la protección en la economía en su conjunto y no en' una industria en particular. Esto no significa que el análisis del equilibrio parcial y del equilibrio general sean incompatibles; sin embargo, el enfoque del equilibrio general es más útil para estudiar la distribución y asignación de recursos. El análisis del equilibrio parcial no proporciona los instrumentos suficientes para este tipo de análisis, excepto cuando se trata de cambios poco significativos.

Durante los últimos años se ha aplicado el análisis de equilibrio general para estudiar las políticas comerciales. Se considerarán tres tipos de enfoques desarrollados en esta área. El primero, Mussa (1974), estudia los efectos de las tarifas arancelarias en la distribución del ingreso en la tradición del teorema de Stolper-Samuelson; el segundo, Black y Taylor (1974), utiliza el modelo de Johansen y, por último, Shoven y Whalley (1972), utilizan el algoritmo de Scarf que analiza los efectos de los impuestos en el equilibrio general.

Para los propósitos de este trabajo, se dedica la siguiente sección a elaborar una breve comparación de estos tres enfoques, con el objeto de mostrar que la flexibilidad y generalidad del tercero compensa sus posibles desventajas. La tercera sección tratará sobre la presentación del algoritmo tal como se aplica al análisis de los efectos de políticas comerciales. Finalmente, se utilizará el algoritmo en la simulación de una economía ficticia

* Este trabajo se realizó para el taller sobre "Development Programming", impartido por los profesores T. Koopmas y J. McCabe en la Universidad de Yale (otoño de 1976). 
y simplificada. El propósito es aplicar este método a un estudio posterior sobre el caso de México.

\section{TRES ENFOQUES: UNA COMPARACIÓN}

Mussa, en su artículo "Tariffs and the Distribution of Income", ${ }^{1}$ analiza los efectos de los cambios, inducidos por las tarifas, en los precios relativos de los bienes en la distribución del ingreso entre los factores de producción. Mussa hace la distinción entre el análisis a corto plazo - se centra en factores específicos y el grado de sustitución entre estos factores y los de movilidad elevada - y el de largo plazo -el cual permite que los factores específicos se asignen a usos alternativos. En el primer modelo, Mussa considera al capital (uno de los dos factores) como un factor específico, mientras que el trabajo se moviliza con toda libertad entre las industrias. En su segundo modelo, Mussa hace el capital un factor cuasifijo; en el corto plazo (capital fijo) este modelo es idéntico al primero, mientras que en el largo plazo el modelo es igual al de Stolper-Samuelson.

Mussa prueba que, dentro del primer modelo, la magnitud de los cambios en el ingreso de los factores depende del grado de sustitución entre ellos $(L, K)$ y de la intensidad en el uso de los factores en ambas industrias. Este resultado se debe, por un lado, a que la elasticidad de la demanda por el trabajo depende de la elasticidad de sustitución entre el trabajo y el capital y de la participación distributiva del trabajo en el valor de la producción. Por otro lado, se debe a que los cambios en el ingreso del capital pueden representarse como una función de la participación del capital en ambas industrias. ${ }^{2}$

Con el objeto de mostrar que existen diferencias en cada uno de los factores entre el plazo largo y el corto, Mussa sugiere que los cambios en el ingreso, en el corto plazo, están determinados totalmente por el supuesto de que el trabajo es móvil mientras que el capital permanece inmóvil. Sin embargo, a largo plazo las magnitudes de los cambios en el ingreso de los factores son independientes del grado de sustitución entre capital y trabajo. Estas diferencias originan conflictos entre los factores. ${ }^{3}$

Estos resultados se apoyan en los siguientes supuestos: $a$ ) que existen dos bienes y tres factores (solamente un factor móvil, el trabajo, se utiliza en la producción de ambos bienes; dos factores específicos: el capital en la producción de los bienes 1 y 2 ); $b$ ) que las funciones de producción para los dos bienes son lineales y homogéneas en sus respectivos insumos y cuentan con todas las características de concavidad; $c$ ) que la cantidad de trabajo utilizado conjuntamente en ambas industrias es igual a la oferta agregada de trabajo.

\footnotetext{
1 Véase la referencia (3).

2 Véase Mussa, p. 1196.

3 Véase Mussa, p. 1202.
} 
Este enfoque tiene ciertas desventajas porque a pesar de que Mussa amplía su análisis a $n$ bienes, para demostrar que las conclusiones más importantes se cumplen aún en este caso, el estudio no tiene grandes posibilidades de aplicación empírica.

Black y Taylor, en "Practical General Equilibrium Estimation of Resources Pulls under Trade Liberalization",4 analizan el problema de determinar la respuesta real de la economía a cambios en políticas comerciales dentro de un esquema de equilibrio general. Los autores utilizan un modelo similar al elaborado por Leif Johansen (A Multisectoral Study of Economic Growth, 1964), que consiste en utilizar una aproximación lineal en logaritmos a la solución del equilibrio general para la economía. Black y Taylor aplican este modelo al caso de Chile, utilizando 35 sectores en donde el trabajo es el único factor de producción variable; esta característica hace que esencialmente el modelo sea de corto plazo.

Las principales conclusiones de su investigación se relacionan con los efectos de la especificación de la forma en que los insumos intermedios entran en la función de producción. Dependiendo de las especificaciones, se dan diferencias numéricas en las respuestas de la producción a los cambios en las tarifas arancelarias.

Al analizar la especificación de coeficientes fijos en las funciones de producción Cobb-Douglas para los bienes finales, los autores llegaron a una fórmula simple de equilibrio parcial.

$$
X_{i}^{1}=\frac{\alpha_{i}}{1-\alpha_{i}}\left(P_{i}^{*}\right)
$$

lo cual significa que el cambio logarítmico de la producción en el sector $i$ es igual al cambio logarítmico en el precio neto del sector (expresado en unidades de salario), multiplicado por su elasticidad de oferta.

En el caso de la especificación Cobb-Douglas

$$
\left(X_{i}=A_{i} L_{i}^{\alpha_{i}} K_{i}^{\beta_{i}} X_{j i}^{\gamma_{j i}}\right)
$$

el cambio logarítmico en la producción del sector está dado por

$$
X_{i}^{\prime}=\alpha_{i} L_{i}^{\prime}+\sum_{j} \gamma_{j i} X_{j i}^{\prime}
$$

que difiere del caso de coeficientes fijos, donde los cambios logarítmicos en el uso intermedio $\left(X_{i j}^{\prime}\right)$ afectan los niveles de producción.

Estos resultados se apoyan en los supuestos que se mencionan a continuación. Primero, que existe una función de utilidad agregada con la propiedad de separabilidad; segundo, que la producción se lleva a cabo bajo

4 Véase referencia (1). 
condiciones de competencia (las distintas especificaciones se describieron con anterioridad). Tercero, que la oferta de importaciones es infinitamente elástica pero que la elasticidad de la demanda por exportaciones es menos que infinita; cuarto, que el único papel que adopta el gobierno es la imposición de impuestos indirectos y tarifas arancelarias; quinto, que las funciones de producción tienen rendimientos decrecientes solamente en un factor: el trabajo (que hace que el modelo sea en esencia de corto plazo). Por último, que todos los bienes importados no se fabrican internamente $o$, en otras palabras, que las importaciones sean no competitivas.

Este modelo también tiene sus limitaciones. Aun cuando predice con exactitud los efectos de la política comercial en la asignación de recursos, no proporciona información sobre los cambios en la distribución del ingreso. También, su naturaleza de corto plazo representa una limitación importante. Más aún, la linearidad de las ecuaciones no siempre representa una especificación adecuada. Sin embargo, este modelo es uno de los pocos intentos por analizar los efectos del equilibrio general de la política comercial. El modelo de Black y Taylor, comparado con el de Mussa tiene un margen más amplio de predicción, dada la simplicidad teórica subyacente de este último.

Shoven y Whalley, en "A General Equilibrium Calculation of the Effects of Differential Taxation of Income from Capital in the U.S.", ${ }^{5}$ hacen uso del algoritmo de Scarf al analizar la incidencia de los efectos y los costos de eficiencia asociadas con la tributación diferencial en el ingreso proveniente del capital en los Estados Unidos. ${ }^{6}$ Los autores utilizan un modelo de equilibrio general que toma en consideración: 1) un conjunto de funciones de demanda de mercado (con $n$ insumos y productos); 2 ) la descripción de las posibilidades de producción a través del listado de actividades disponibles; y 3 ) el acervo inicial de bienes en la economía. E1 total de la demanda de mercado es la suma de demandas individuales (derivadas de la maximización de la utilidad). El equilibrio competitivo se caracteriza porque la oferta es igual a la demanda y todas las actividades generan cero ganancias. Al introducir los impuestos los autores utilizan un bien ficticio: "boletos de impuestos sobre el capital". Bajo esta técnica se analizan los efectos de la tributación comparando los resultados en los modelos con y sin boletos. ${ }^{7}$

Dados los supuestos de este enfoque, es conveniente concluir que la aplicación del algoritmo de Scarf no precisa de cálculos diferenciales; que no son necesarios supuestos de linearidad (o uso de expresiones logarítmicas, como en el modelo de Black y Taylor); que no es restrictivo en el

5 Véase referencia (5).

${ }^{6}$ Los autores analizan la tributación al capital en los Estados Unidos y comparan sus resultados con los obtenidos por Harberger ("The Incidence of the Corporation Income Tax", en Journal of Political Economy 70, 215-240). Shoven y Whalley muestran que los resultados de Harberger son un caso especial de los suyos.

? Para una descripción detallada de esta técnica, ver la sección siguiente. 
número de factores de producción (como el de Mussa); que se toman en consideración varios consumidores con distintos gustos y dotaciones iniciales; que el modelo puede aplicarse al análisis de corto y largo plazos. Este modelo, además de su eficiencia en el cálculo como el de Black y Taylor, también proporciona información sobre los efectos de la distribución del ingreso entre individuos y entre factores.

\section{TARIFAS ARANCELARIAS EN EL MODELO DE EQUilibrio GeNERAL:} EL ALGORITMO DE SCARF

\section{El algoritmo}

Este algoritmo puede resumirse de la siguiente manera: ${ }^{8}$

i) Para que el algoritmo trabaje es necesario que las funciones de demanda de mercado satisfagan la ley de Walras:

$$
\sum_{i=1}^{n} P_{i}\left(X_{i}-W_{i}\right)=0
$$

donde $\quad X_{i}=\sum_{j}^{j}{ }_{i}^{j} X_{i j}=$ demanda de mercado para el bien $i$.

$$
W_{i}={ }_{j} \underline{\Sigma}_{1}^{j} w_{i j}=\text { total de dotaciones iniciales del bien } i \text {. }
$$

La expresión (1) establece que el valor del exceso de demanda del mercado es cero.

ii) La producción se describe por medio de una matriz de análisis de actividades $A$ en donde las columnas representan actividades que se utilizan a un nivel no negativo (estas columnas incluyen las actividades de disposición libres). Se supone que la frontera de posibilidades de producción es finita en todas sus dimensiones.

iii) El equilibrio competitivo se define por un vector de precios $P^{*} \mathrm{y}$ un vector de niveles de actividad $Y^{*}$ tal, que:

$$
\begin{aligned}
& X_{i}\left(P^{*}\right)=W_{i}+\sum_{j=1}^{m} a_{i j} Y_{j}^{*} \\
& \text { y } \quad \sum_{j} P_{i} a_{i j} \leq 0 \chi_{j}\left(=\operatorname{si} Y_{j}^{*}>0\right)^{9}
\end{aligned}
$$

8 Para una descripción completa ver Scarf, referencia (1).

${ }^{9}$ Las funciones de demanda son homogéneas de grado cero con respecto a los precios; por lo tanto, resulta válido normalizar:

$$
\stackrel{\sum}{i}_{1}^{n} P_{i}=1, P_{i} \geqq 0
$$


El objetivo del algoritmo es encontrar $P^{*}$ y $Y^{* 10}$ que satisfagan las condiciones descritas. Para este fin, se define una matriz $B\left(b_{i j}\right)$ cuyas columnas corresponden a los vectores de precios $P^{1}, \ldots, P^{k}$; cada vector de precios $P^{i}$ está asociado con la columna correspondiente de vectores de la matriz $B, b^{i}$. La relación entre $P^{i}$ y $b^{i}$ puede resumirse de la siguiente forma:

a) Si alguno de los elementos de $P^{i}$ es cero, entonces $b^{i}$ contiene un 1 en lugar del primer precio cero y ceros en el resto.

b) Si se simboliza la actividad con la mayor ganancia ( $\left.\Pi^{*}\right)$ como $a^{*}$ :

Si $\Pi^{*}>0, b^{i}=-a^{*}$

Si $\Pi^{*}<0, b^{i}=\times\left(P^{i}\right)$

$i v)$ Si se define un subconjunto de $n$ precios de la lista $P^{1}, \ldots, P^{k}$, como el conjunto primitivo, puede establecerse el principal teorema en que se apoya el algoritmo.

Existe un conjunto primitivo $P^{j 1}, \ldots, P^{j n}$ tal que $B Y \equiv W$ tiene una solución no negativa donde $Y_{j}=0$ para $j \neq j_{1}, \ldots, j_{n}$.

\section{Introducción de tarifas arancelarias en el algoritmo}

La idea de introducir impuestos a través de la creación de un bien ficticio ("boleto") fue original de Shoven y Whalley (1974). Tal como se mencionó en la breve descripción de su modelo, los autores se preocupan por el análisis de los efectos de equilibrio general de los impuestos en el ingreso proveniente del capital. Se adoptará esta técnica para analizar las tarifas arancelarias.

La preocupación en este trabajo se refiere a distorsiones ad valorem; las tarifas arancelarias representan imposiciones ad valorem aplicados a las importaciones. Se incluirán las tarifas arancelarias en el modelo general por medio de la compra de boletos por los agentes que importan bienes. En otras palabras, cualquier agente que importe bienes o factores debe comprar un cierto número de boletos por unidad del bien importado. Esto puede representarse de la siguiente manera: si la importación del bien $X$ debe pagar impuestos a una tasa $\gamma$, el agente objeto de impuesto debe comprar $\gamma(P x / P t)$ boletos $(t)$ conjuntamente con cada unidad de $X$ (Pt y $P x$ son los precios de los boletos y del bien $X$, respectivamente).

Una vez encontrado el número de boletos — dada la tasa - a comprarse por cada unidad de bien importado, se enfrenta otro problema: la forma de utilización de esta técnica para encontrar los efectos de las tarifas arancelarias en la distribución y asignación de recursos. La introducción de las tarifas arancelarias no es más que la introducción de un nuevo bien en la matriz de análisis de actividades. Este nuevo bien aparecerá con signo negativo en todas las actividades de importación sujetas a esta obligación.

${ }_{10}$ Se crea un grid de vectores de precios $P^{n+1}, \ldots, P^{k}$ en el simplex unitario. 


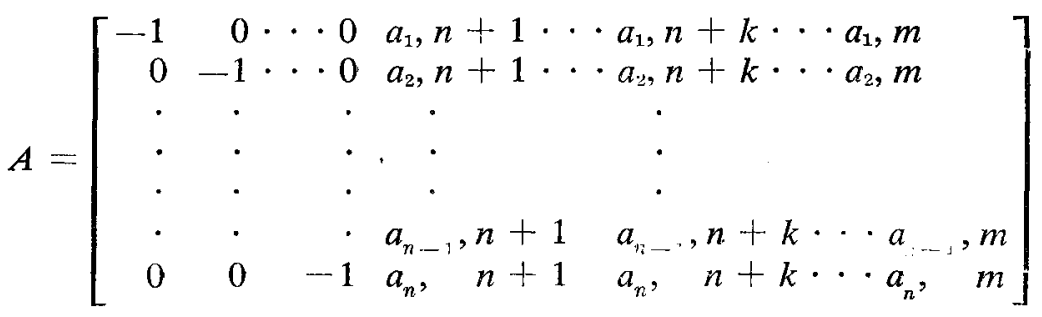

Esta matriz de análisis de actividades $(A)$ representa $m$ procesos distintos que podrían utilizarse en la producción de $n$ bienes. Supóngase que los procesos $a_{1}, n+1$ hasta $a_{1}, n+k$ corresponden a importaciones $\mathrm{y}$ que el gobierno desea imponer tarifas en todas éstas. ${ }^{11} \mathrm{La}$ introducción de tarifas arancelarias es tan simple como que el último renglón de la matriz sea el del boleto. Los valores de los distintos coeficientes dependerán de las distintas tasas. Por ejemplo, si los primeros $g$ procesos de importación están sujetos a una tarifa de $\delta$ en términos relativos, y el resto a una tarifa de $\gamma$ por ciento, entonces:

$$
\begin{aligned}
& A n, n+1=\delta\left(P_{i} / P_{t}\right), \ldots, A n, n+1+g=-\delta\left(P_{h} / P_{t}\right), \mathrm{y} \\
& A n, n+1+g+1=-\gamma\left(P_{c} / P_{t}\right), \ldots, A n, n+k=- \\
& \quad \gamma\left(P_{j} / P_{t}\right), \text { etc. } .
\end{aligned}
$$

donde el proceso $n+1$ produce el bien $i$ (o sea las importaciones), por lo que el elemento $a_{i}, n+1$ debe ser igual a la unidad ${ }^{12}$ y así para todos.

\section{Análisis de los efectos de las tarifas arancelarias}

Una vez introducidos los boletos surge la pregunta sobre el uso de esta técnica para analizar los efectos de distribución y asignación. El mecanismo de la técnica es tan sencillo como la simple introducción de los boletos. Deben utilizarse dos matrices distintas de análisis de actividades: la matriz $A$ que ya se presentó y la matriz $A^{1}$ cuya diferencia más importante respecto de la matriz $A$ es que no cuenta con la enésima columna (o sea la última actividad de disposición libre) ni con el enésimo renglón (boletos).

Al resolver los dos problemas diferentes se obtienen dos conjuntos distintos de precios de equilibrio, con y sin tarifas arancelarias; si se comparan estos dos conjuntos pueden encontrarse los efectos de las tarifas arance-

11 Obsérvese que esta técnica permite tomar en consideración distintos niveles de tarifas arancelarias.

12 Como se mencionó, $\gamma\left(P_{i} / P_{t}\right)$ es el número de boletos que debe comprar el agente objeto de tributación junto con cada unidad del bien $i$ que se importa. Es evidente -como se verificará en la siguiente sección- que si la matriz de análisis de actividades no presenta la unidad en el coeficiente de producción, lo que entrará en la matriz será si se importan $\lambda$ unidades del producto $i$, entonces se utilizará $=\lambda \gamma\left(P_{i} / P_{t}\right)$. 
larias en la distribución del ingreso y asignación de recursos. ${ }^{13}$ Este último paso no es tan claro como parece. Se intentará explicar con más detalle las condiciones necesarias para efectuar esta comparación:

i) Definir las posibilidades de producción para cada sector y aproximar las funciones de producción utilizando un número dado de actividades.

ii) Definir un número determinado de consumidores y considerarlos entonces heurísticamente como representativos de distintos receptores del ingreso (ver la siguiente sección). Cada individuo $j$ tendrá una dotación inicial del bien $i\left(W_{i j}\right)$ y el total de la dotación del individuo será $\sum_{i=1}^{n} W_{i j}$, por lo que su ingreso está dado por $I_{j}=\sum_{i} \sum_{1}^{n} P_{i} W_{i j}$ (ingreso del consumidor $j$ ).

iii) Definir las funciones de demanda para cada individuo:

$X_{i j}=f\left(P_{i}, I_{j}\right)$ (demanda del consumidor $j$ por el bien $i$ ); ésta es una función del precio del bien y del ingreso del individuo.

iv) Pueden resolverse dos casos distintos sin cambiar las características del problema; los casos con y sin tarifas arancelarias pueden arrojar diferentes precios del equilibrio; distintos totales de demanda por los bienes; diversas participaciones relativas de los factores; demandas individuales distintas para cada bien; participaciones relativas del individuo diferentes y distinto producto nacional bruto. Estas consideraciones quedarán más claras en el ejemplo siguiente.

\section{EJEMPLO: UNA ECONOMf́A FICTICIA SIMPLIFICADA}

En este ejemplo se supone, heurísticamente, que existen tres consumidores: el 1, que representa el 10\% de los receptores del ingreso de la economía (que, dadas las dotaciones iniciales, podrían denominarse capitalistas y latifundistas); consumidor 2, que representa el $90 \%$ de la parte restante (los trabajadores), y el consumidor 3 que es el gobierno. Estos consumidores están dotados de cierta cantidad de bienes existentes en el inicio del periodo.

Se partirá del supuesto de que es una economía abierta y que todas las importaciones son competitivas. Al mismo tiempo, se supone una situación de "país pequeño", los precios de los bienes que se importan se toman como dados.

\section{a) Sin politica comercial}

$\mathrm{Al}$ analizar el caso donde no existen tarifas arancelarias, se tiene una economía con cinco bienes o productos:

$$
\begin{aligned}
& 1=\text { capital }(K) \\
& 2=\text { trabajo }(L) \\
& { }_{13} \text { Se supone que el equilibrio es único. }
\end{aligned}
$$


$3=$ bienes manufacturados $(M)$

$4=$ bienes agrícolas $(A)$

$5=$ divisas $(F)$

Las posibilidades de producción de esta economía se presentan en la matriz 1 de análisis de actividades:

Matriz 1

\begin{tabular}{rrrrrrrrrr}
\hline & 6 & 7 & 8 & 9 & 10 & 11 & 12 & 13 \\
\hline (K) & 1 & -1.5 & -1.0 & -2.0 & -0.8 & -0.03 & -0.01 & -0.03 & -0.01 \\
(L) & 2 & -1.0 & -2.5 & -0.0 & -2.0 & -0.05 & -0.02 & -0.05 & -0.02 \\
(L) & 3 & 2.0 & 1.0 & 2.5 & 0.0 & 4.00 & 0.00 & 4.00 & 0.00 \\
(A) & 4 & 1.0 & 2.0 & 0.0 & 3.5 & 0.00 & 4.00 & 0.00 & 4.00 \\
(F) & 5 & 0.0 & 0.0 & 0.0 & 0.0 & -1.50 & -1.00 & 1.50 & 1.00 \\
\hline
\end{tabular}

No se incluyen las actividades de disposición libre. Las primeras cuatro actividades $(6,7,8,9)$ representan los procesos internos: la primera produce, conjuntamente, bienes manufacturados y agrícolas, como sucede con al segunda (nótese que en la actividad 6 la producción de bienes manufacturados equivale a dos tantos la producción de bienes agrícolas, mientras que en la actividad 7 se da el caso contrario; también obsérvese que la actividad 6 utiliza más capital y menos trabajo que la actividad 7). Las actividades 8 y 9 producen bienes manufacturados y agrícolas, respectivamente (el uso del capital en la actividad 8 es mayor que en la actividad 9; esto hace que en términos relativos los bienes agrícolas sean de uso intensivo en mano de obra). Las últimas cuatro actividades representan los procesos de importación $(10,11)$ y exportación $(12,13)$. Obsérvese que el último renglón, divisas, está conformado a modo de tomar los precios internacionales como dados. Por ejemplo, el proceso 10 representa un proceso de importación de 4 unidades de bienes manufacturados; el proceso 11 importa 3 unidades de bienes de origen agrícola, mientras que los procesos 12 y 13 representan exportaciones de estos artículos.

Pasemos al lado de la demanda de la economía. Se parte del supuesto de que los tres consumidores maximizan su utilidad y, en virtud de que sus funciones de utilidad muestran una elasticidad de sustitución constante (CES), se obtienen las siguientes funciones de demanda:

$$
\begin{aligned}
D 1 & =\left[a_{11} F_{1}(\Pi) / \Pi_{1}^{b_{1}}, \ldots, a_{15} F_{5}(\Pi) / \Pi_{5}^{b_{1}}\right] \\
D 2 & =\left[a_{21} F_{1}(\mathrm{II}) / \Pi_{1}^{b_{2}}, \ldots, a_{25} F_{5}(\Pi) / \Pi_{5}^{b_{2}}\right] \\
D 3 & =\left[a_{31} F_{1}(\Pi) / \Pi_{1}^{b_{3}}, \ldots, a_{35} F_{5}(\Pi) / \Pi_{5}^{b_{3}}\right]
\end{aligned}
$$


Los consumidores demandarán $D_{1}, D_{2}$ y $D_{3}$ a los precios de $\Pi_{i}$, respectivamente; $a_{i j}$ describe la intensidad de la demanda de los consumidores por el bien $j$; y $b_{i}$ describe la elasticidad de sustitución entre bienes del i-ésimo consumidor.

Las intensidades $a_{i j}$ están dadas por la siguiente información:

\begin{tabular}{|c|c|c|c|c|c|}
\hline & \multicolumn{4}{|c|}{ Bienes } & \multirow[b]{2}{*}{5} \\
\hline & 1 & 2 & 3 & 4 & \\
\hline Consumidor 1 & $\begin{array}{l}0 \\
0 \\
0\end{array}$ & $\begin{array}{l}0 \\
0 \\
0\end{array}$ & $\begin{array}{l}0.8 \\
0.2 \\
0.5\end{array}$ & $\begin{array}{l}0.2 \\
0.8 \\
0.5\end{array}$ & $\begin{array}{l}0 \\
0 \\
0\end{array}$ \\
\hline
\end{tabular}

Este cuadro refleja que no existe demanda por los bienes 1,2 y 5 (capital, trabajo y divisas). También refleja que los "capitalistas y latifundistas" demandan más, en términos relativos, los bienes manufacturados, mientras que los "trabajadores" se dirigen más hacia los de origen agrícola. Por su parte, el gobierno demanda con la misma intensidad todos los bienes.

Las elasticidades respectivas están dadas por las cifras siguientes.

\begin{tabular}{rl} 
& $b_{i}$ \\
\hline Consumidor 1 & 2.0 \\
2 & 1.5 \\
3 & 0.5 \\
\hline
\end{tabular}

Al mismo tiempo, estos consumidores cuentan con cierta dotación inicial, según se muestra en el siguiente patrón de propiedad:

\begin{tabular}{c|cccccc} 
& & $1(K)$ & $2(L)$ & $3(M)$ & $4(A)$ & $5(F)$ \\
\hline Consumidor 1 & 5.0 & 1 & 0 & 0 & 0 \\
2 & 0.4 & 9 & 0 & 0 & 0 \\
3 & 0.5 & 1 & 0 & 0 & 0 \\
\hline
\end{tabular}

Como ya se mencionó, el consumidor 1 tiene en su haber una gran proporción del total de la economía a la vez que los trabajadores cuentan con la proporción más elevada del total del trabajo; el gobierno posee proporciones pequeñas de ambos factores.

Si se utiliza el algoritmo de Scarf, ${ }^{14}$ explicado en la sección anterior, se obtienen los resultados finales para esta economía, que proporcionan en el cuadro A. De ahí se deriva que la actividad 6, que produce conjuntamente bienes manufacturados y de origen agrícola, se utiliza a nivel de 1.35;

14 Para este ejemplo, el tamaño del grid es de 500; los precios se normalizan por el tamaño del grid. El número total de iteraciones fue de 1818. 
la actividad 9, que produce exclusivamente bienes de origen agrícola, a nivel de 4.79; la actividad 10 (importación de bienes manufacturados) a nivel de $1.09 \mathrm{y}$, por último, la actividad 13, que exporta bienes de origen agrícola, a nivel de 1.64. Esta economía ficticia produce bienes manufacturados y agrícolas, exportando estos últimos e importando bienes manufacturados.

En el cuadro 1 también se observa que los capitalistas y latifundistas. poseen la proporción más elevada de bienes manufacturados, mientras que los trabajadores cuentan con la proporción más elevada de los bienes agrícolas. El gobierno posee la proporción más baja de ambos..$^{15}$ También se observa que el precio del capital es mayor que el precio del trabajo $\left(\frac{w}{r}=\frac{0.09}{0.22}\right) ;$ dadas las dotaciones de trabajo y capital de los distintos consumidores, pueden calcularse sus respectivos ingresos:

$$
\text { Ingresos del consumidor } \begin{aligned}
1 & =1.20 \\
2 & =0.90 \\
3 & =0.2^{16}
\end{aligned}
$$

No hay duda de que los "capitalistas y latifundistas" poseen la participación mayor. En otras palabras, el 10\% más elevado de los receptores del ingreso de la sociedad perciben el $52 \%$ del total de la producción. ${ }^{17}$ Por otro lado, es evidente que los consumidores gastarán todos sus ingresos. ${ }^{18}$

En el cuadro 1 se encuentran los totales de trabajo y capital utilizados en cada actividad y, por consiguiente, en la economía en su conjunto. La actividad 6 utiliza 1.5 unidades de capital y 1 unidad de trabajo; los totales de capital y trabajo en esta actividad son de 2.025 y 1.35 , respectivamente. Siguiendo el mismo criterio en todas las actividades, se observa que la razón capital/trabajo es de 5.89/11.01. Si se multiplican por sus precios respectivos, se obtiene un cociente de las participaciones de capital y trabajo: $\frac{r K}{w L}=\frac{1.3}{0.99}$. No puede establecerse una relación entre la distribución del

15 Pueden existir distintas interpretaciones de los diversos usos que el gobierno hace con los bienes. No es el propósito de este trabajo discutir las políticas de distribución pero sí analizar los efectos de la política comercial.

16 Por ejemplo, $1.2=r K_{1}+w L_{1}=(0.22)(5)+(0.09)$ (1), donde $K_{1}$ y $L_{1}$ representan la dotación de trabajo y capital para el consumidor 1.

17 En este caso, el gobierno es una "institución neutral"; el ingreso proviene exclusivamente de sus dotaciones iniciales provenientes del capital y trabajo. En el caso siguiente —l de política comercial- el gobierno efectúa una intervención, la que se refleja en que es el único propietario de los boletos.

18 Consumidor $1=P_{M}\left(M_{1}\right)+P_{A}\left(A_{1}\right)=1.18 \approx 1.2$

Consumidor $2=P_{M}\left(M_{2}\right)+P_{A}\left(A_{2}\right)=0.90=0.9$

Consumidor $3=P_{M}\left(M_{3}\right)+P_{A}\left(A_{3}\right)=0.21 \approx 0.2$

donde $M_{i}$ y $A_{i}$ son las asignaciones finales de los bienes manufacturados y de origen agrícola en manos del consumidor $i$. 
ingreso personal y funcional puesto que los capitalistas y latifundistas poseen cierta cantidad de trabajo y los trabajadores cuentan con capital.

\section{b) Politica comercial}

En este caso, la economía ficticia cuenta con siete bienes o productos:

1. capital $(K)$

2. trabajo $(L)$

3. bienes manufacturados $(M)$

4. bienes de origen agrícola $(A)$

5. boletos $1\left(T_{1}\right)$

6. boletos $2\left(T_{2}\right)^{19}$

7. divisas $(F)$

Las posibilidades de producción de la economía están dadas por la matriz 2 de análisis de actividades, que es idéntica a la anterior, con excepción de los renglones 5 y 6 .

Matriz 2

\begin{tabular}{lrrrrrrrrr}
\hline & 8 & 9 & 10 & 11 & 12 & 13 & 14 & 15 \\
\hline (K) & 1 & -1.5 & -1.0 & -2.0 & -0.8 & -0.03 & -0.01 & -0.03 & -0.01 \\
$(\mathrm{~L})$ & 2 & -1.0 & -2.5 & -0.8 & -2.0 & -0.05 & -0.02 & -0.05 & -0.02 \\
$(\mathrm{M})$ & 3 & 2.0 & 1.0 & 2.5 & 0.0 & 4.00 & 0.00 & -4.00 & 0.00 \\
$(\mathrm{~A})$ & 4 & 1.0 & 2.0 & 0.0 & 3.5 & 0.00 & 4.00 & 0.00 & -4.00 \\
$\left(\mathrm{~T}_{1}\right)$ & 5 & 0.0 & 0.0 & 0.0 & 0.0 & -0.20 & 0.00 & 0.00 & 0.00 \\
$\left(\mathrm{~T}_{2}\right)$ & 6 & 0.0 & 0.0 & 0.0 & 0.0 & 0.00 & -0.10 & 0.00 & 0.00 \\
$(\mathrm{~F})$ & 7 & 0.0 & 0.0 & 0.0 & 0.0 & -1.50 & -1.00 & 1.50 & 1.00 \\
\hline
\end{tabular}

Las actividades 12 y 13 (equivalentes a las 10 y 11 de la primera matriz) representan la única diferencia entre las dos matrices; las diferencias se expresan en los renglones 5 y 6 . En el renglón 5 se obtiene un coeficiente - 0.20 que representa el número necesario de boletos para importar 4 unidades de productos manufacturados. Se supone que los precios de los boletos son iguales a los precios internacionales dados del bien importado. Por lo tanto, el coeficiente -0.20 representa una tarifa arancelaria de $5 \%$ sobre las importaciones de bienes manufacturados. El coeficiente -0.10 representa una tarifa arancelaria de $2.5 \%$ en las importaciones de bienes de origen agrícola. ${ }^{20}$

19 Tal como se mencionó en la sección anterior, los agentes de la economía que importan bienes deben comprar boletos. Estos últimos representan tarifas arancelarias: $T_{1}$ representa la tarifa impuesta a las importaciones de bienes manufacturados y $T_{2}$ a la de artículos de origen agrícola.

${ }_{20}$ Obsérvese que se tienen dos actividades más de disposición libre y que la actividad 8 corresponde a la actividad 6 de la matriz anterior y así sucesivamente. 
En el lado de la demanda se tienen exactamente las mismas condiciones que en el primer caso, porque la demanda por los boletos tiene la intensidad de cero para todos los consumidores $\mathrm{y}$, por lo tanto, no ha sufrido ningún cambio. El patrón de propiedad está dado en el siguiente cuadro:

\begin{tabular}{r|ccccccc} 
& $1(\mathrm{~K})$ & $2(\mathrm{~L})$ & $3(\mathrm{M})$ & $4(\mathrm{~A})$ & $5\left(\mathrm{~T}_{1}\right)$ & $6\left(\mathrm{~T}_{2}\right)$ & $7(\mathrm{~F})$ \\
\hline Consumidor 1 & 5 & 1 & 0 & 0 & 0 & 0 & 0 \\
2 & 0.4 & 9 & 0 & 0 & 0 & 0 & 0 \\
3 & 0.5 & 1 & 0 & 0 & 0.15 & 0.10 & 0 \\
\hline
\end{tabular}

El uso del algoritmo ${ }^{21}$ proporcionó los resultados que se presentan en el cuadro 1 . Se observa que la razón precio del capital-precio del salario disminuye de 0.4 a 0.08 . Al mismo tiempo, se da un descenso en los demás precios en términos absolutos. ${ }^{22}$ También puede observarse que el nivel de actividad 8 sufre un aumento de 1.35 a 1.36; que la nueva actividad 9 en uso produce 2 unidades de bienes agrícolas y 1 unidad de bienes manufacturados, utilizando más mano de obra que capital. La actividad 11 sufre un descenso en su nivel de producción; la actividad 12 —importaciones- disminuye su nivel de producción de 1.09 a 0.75 (las importaciones descienden sustancialmente debido a la política comercial $(\gamma=5 \%)$; por último, se observa un descenso en el nivel de "producción" de las exportaciones de 1.64 a 1.13 . Sin embargo, la economía sigue produciendo bienes manufacturados y de origen agrícola, a la vez que importa bienes manufacturados y exporta los de origen agrícola. La oferta total de bienes manufacturados sufrió un descenso pero se generó un incremento en la oferta total de bienes agrícolas. Esto no significa que la producción interna de bienes manufacturados haya sufrido un descenso, muy por el contrario, se registró un aumento de 2.70 a 3.31. Como resultado de la política comercial, se da un incremento en la producción interna del bien protegido.

En términos de asignación final, en el cuadro 1 se observa que el consumidor 3 mejora mientras que el consumidor 2 observa un deterioro en su situación; el consumidor 1 cuenta con un número menor de bienes manufacturados y uno mayor de bienes de origen agrícola.

Dado el patrón de propiedad, los ingresos de los tres consumidores están dados por la siguiente información:

$$
\text { Consumidor } \begin{aligned}
1 & =0.66 \\
2 & =0.142 \\
3 & =0.16
\end{aligned}
$$

21 El tamaño del grid es el mismo (500). El número de iteraciones fue de 14914.

22 Obsérvese que $P_{M}=(1+\gamma) P_{F} P_{M}^{w}$; donde $P_{M}^{w}$ representa el precio mundial de bienes manufacturados y que es igual al precio de los boletos. 
A pesar del descenso en el total de la producción, la participación relativa del consumidor 1 observa un incremento de $52 \%$ a $69 \%$ : el $10 \%$ más elevado de los receptores de ingreso obtiene mayores ingresos en términos relativos, dado que la política comercial ha hecho la distribución del ingreso más desigual. ${ }^{23}$

En el cuadro 1 puede analizarse el lado de la producción y se observa que la actividad 9 se utiliza a nivel positivo, lo que significa una reasignación de factores. Como se mencionó, la oferta total de bienes de origen agrícola sufrió un aumento mientras que la oferta total de bienes manufacturados descendió. Por lo tanto, no es sorprendente que el cociente de participaciones relativas del capital y trabajo haya aumentado: $r k / w L=0.77 / 0.10$. De nuevo, no puede analizarse la relación entre la

\section{Cuadro 1}

\begin{tabular}{|c|c|c|c|c|}
\hline & \multicolumn{2}{|c|}{$\begin{array}{l}\text { Sin } \\
\text { politica comercial }\end{array}$} & \multicolumn{2}{|c|}{$\begin{array}{c}\text { Con } \\
\text { polftica comercial }\end{array}$} \\
\hline \multicolumn{5}{|l|}{ Precios } \\
\hline$r$ & \multicolumn{2}{|c|}{0.22} & \multicolumn{2}{|c|}{0.13} \\
\hline พ & \multicolumn{2}{|c|}{0.09} & \multicolumn{2}{|c|}{0.01} \\
\hline $\mathrm{P}_{M}$ & \multicolumn{2}{|c|}{0.16} & \multicolumn{2}{|c|}{0.08} \\
\hline$P_{A}$ & \multicolumn{2}{|c|}{0.10} & \multicolumn{2}{|c|}{0.03} \\
\hline $\mathrm{P}_{F}$ & \multicolumn{2}{|c|}{0.42} & \multicolumn{2}{|c|}{0.14} \\
\hline $\mathrm{PT}_{1}=\mathrm{PM}_{\mathrm{W}}^{\mathrm{N}}$ & \multicolumn{2}{|c|}{0.00} & \multicolumn{2}{|c|}{0.60} \\
\hline $\mathrm{PT}_{2}$ & \multicolumn{2}{|c|}{0.00} & \multicolumn{2}{|c|}{0.00} \\
\hline & \multicolumn{2}{|c|}{ Nivel } & \multicolumn{2}{|c|}{ NiveI } \\
\hline \multicolumn{5}{|l|}{ Actividades } \\
\hline 6 y 8 & \multicolumn{2}{|c|}{1.35} & \multicolumn{2}{|c|}{1.36} \\
\hline $7 \times 9$ & \multicolumn{2}{|c|}{0.00} & \multicolumn{2}{|c|}{0.89} \\
\hline 9 y 11 & \multicolumn{2}{|c|}{4.79} & \multicolumn{2}{|c|}{4.07} \\
\hline 10 y 12 & \multicolumn{2}{|c|}{1.09} & \multicolumn{2}{|c|}{0.75} \\
\hline 13 y 15 & \multicolumn{2}{|c|}{1.64} & \multicolumn{2}{|c|}{1.13} \\
\hline \multicolumn{5}{|l|}{ Oferta (=Demanda) } \\
\hline Articulo 3 (M) & \multicolumn{2}{|c|}{7.08} & \multicolumn{2}{|c|}{6.33} \\
\hline Articulo 4 (A) & \multicolumn{2}{|c|}{11.54} & & \\
\hline Asignación final & Bien 3 & Bien 4 & Bien 3 & Bien 4 \\
\hline Consumidor 1 & 5.39 & 3.18 & 4.88 & 6.88 \\
\hline Consumidon 2 & 0.95 & 7.49 & 0.24 & 3.54 \\
\hline Consumidor 3 & 0.71 & 0.88 & 1.20 & 1.85 \\
\hline Participaciones re & & & & \\
\hline$r K / W L$ & 0.13 & .99 & & .10 \\
\hline
\end{tabular}

${ }^{23}$ Es muy importante la redistribución del ingreso gubernamental, porque los efectos de la política comercial pueden compensarse con la política redistributiva; nada garantiza lo que se hará en tal distribución. Sin embargo, este tema está fuera de los propósitos de este trabajo. 
distribución del ingreso personal y funcional; podría decirse que los trabajadores que no poseen capital sufren un deterioro mientras que los capitalistas que no cuentan con trabajo obtienen una mejoría neta.

La protección de los bienes intensivos en el uso de capital (bienes manufacturados) da como resultado un aumento en la participación relativa del capital.

En resumen, la política comercial ocasiona un aumento en la participación del $10 \%$ más elevado de los receptores de ingreso (o sea los principales propietarios del capital) de $52 \%$ a $69 \%$, originando una distribución del ingreso más desigual en la sociedad. En términos de la distribución del ingreso funcional, puede concluirse que la protección de la industria intensiva en el uso de capital (el único bien importado de esta economía) aumenta la participación relativa del capital con respecto al trabajo. Este ejemplo también muestra un aumento en la producción interna del bien que se protege, mientras que la oferta total disminuye. Por otro lado, la oferta total de bienes de origen agrícola (el único bien de exportación en esta economía) sube y, finalmente, las actividades de importación y exportación sufren un descenso en su nivel de uso. ${ }^{24}$

\section{Conclusiones}

Con este simple ejemplo se ha mostrado la gran flexibilidad y generalidad del algoritmo de Scarf. Al utilizar la técnica de los boletos, que representa la introducción de la política comercial, el algoritmo permite estudiar los efectos de esta política en la distribución del ingreso personal y funcional, así como su influencia en la asignación de recursos.

En contraste con los enfoques descritos en la segunda sección, este modelo es menos restrictivo en términos de los supuestos que deben efectuarse. $\mathrm{Al}$ mismo tiempo, permite trabajar con un número más elevado de consumidores, bienes y sectores. La información necesaria es razonablemente accesible: por el lado de la producción, se precisa de una matriz de análisis de actividades; por el lado de la demanda, se necesita un conjunto de funciones de demanda para los consumidores y sus dotaciones iniciales. Las ventajas de este enfoque son evidentes: por un lado, los supuestos no son tan restrictivos como en el enfoque de Mussa y, por el otro, su aplicabilidad, así como sus posibilidades de cómputo son muy distintas a las del modelo de Mussa. Por último, las ventajas más importantes sobre el enfoque de Black y Taylor son, en primer lugar, que el algoritmo de Scarf no tiene que ser un modelo a corto plazo y que, en segundo lugar, este algoritmo proporciona información sobre la distribución del ingreso personal y funcional.

${ }^{24}$ Se podrían emitir más enunciados, interpretando los resultados de este ejemplo; por razones de espacio debe limitarse al análisis anterior. 
SERRA: DISTRIBUCIÓN DEL INGRESO Y ASIGNACIÓN DE RECURSOS 261

Por último, debe utilizarse un programa más sofisticado en el análisis de las políticas comerciales reales. Si se trabaja con el mismo espíritu que en el ejemplo, este programa podría ayudar al análisis de los efectos de la política comercial en el caso de México, objetivo final del trabajo. 